\begin{abstract}
AIms: The anti-neutrophilic cytoplasmatic autoantibody-associated vasculitides (AASV) are diseases of relapsing-remitting inflammation. Here we explore the cytokine profile in different phases of disease, looking for pathogenic clues of possible prognostic value.

Results: Interleukin (IL)-6, IL-8 and IL-10 were significantly elevated in plasma. Patients in the stable phase who subsequently developed adverse events had higher IL-8 values. Patients in the stable phase who relapsed within 3 months had lower IL-10 values and higher IL-6 levels.

Conclusions: Patients with AASV have raised circulating cytokine levels compared with healthy controls, even during remission. Raised IL-8 seems associated with poor prognosis. Lower levels of IL-10 and higher levels of IL-6 herald a greater risk of relapse. Patients with systemic vasculitis in clinical remission have persistent disease activity, kept under control by inhibitory cytokines.
\end{abstract}

Key words: Vasculitis, Interleukin-6, Interleukin-8, Interleukin-10, Anti-neutrophilic cytoplasmatic autoantibodies, Inflammation, Autoimmunity

\section{Circulating cytokine profile in anti- neutrophilic cytoplasmatic autoantibody-associated vasculitis: prediction of outcome?}

\author{
Sophie Ohlsson ${ }^{1, C A}$, Jörgen Wieslander ${ }^{1,2}$ and \\ Mårten Segelmark ${ }^{1}$
}

${ }^{1}$ Department of Nephrology, Lund University, Sweden; ${ }^{2}$ Wieslab AB, Lund, Sweden

${ }^{\mathrm{CA}}$ Corresponding Author
Tel: +46462220701
Fax: +46462114356
E-mail: Sophie.Ohlsson@njur.lu.se

\section{Introduction}

Anti-neutrophilic cytoplasmatic autoantibody (ANCA)associated small vessel vasculitis (AASV) is a group of inflammatory disorders, characterized by inflammation and necrosis of blood vessels and frequently granuloma formation. ${ }^{1}$ These patients make autoantibodies against proteins present in the granules of neutrophils and monocytes, mainly proteinase 3 (PR3) and myeloperoxidase (MPO). ${ }^{2}$ The disorders, including Wegener's granulomatosis (WG) and microscopic polyangiitis, have a relapsing-remitting progression and a high mortality if untreated. The pathogenesis is still poorly understood. Proinflammatory cytokines, such as tumor necrosis factor (TNF), interleukin (IL)-1 $\beta$, and IL-8, have been found to be elevated systemically and locally at inflammatory sites in WG. ${ }^{3-6}$ Moreover, a pathogenic role has been attributed to IL-8 in ANCA-associated glomerulonephritis. $^{4}$

As the ANCA titer correlates well with disease activity in some studies, ${ }^{7,8}$ a pathogenic role for these autoantibodies has been implicated. In vitro studies have shown ANCA-induced activation of neutrophils as well as monocytes. ${ }^{2}$ In vitro studies on monocytes have shown increased release of IL-8, MCP-1, TNF- $\alpha$, IL-1 $\beta$, IL- 6 and thromboxane $\mathrm{A}_{2}$ upon stimulation with ANCAs. ${ }^{9-13}$ These studies have been exclusively performed on peripheral blood monocytes isolated from healthy donors. Monocytes are an integral part of granulomas and glomerular crescents in active
AASV, ${ }^{1,12}$ and are therefore of importance in understanding the pathogenesis of AASV. The extent of monocyte activation, measured by soluble products of monocyte activation such as neopterin and IL-6, has in one study been found to correlate with the disease activity in WG. ${ }^{13}$

In this study, our aim was to further elucidate the circulating cytokine profile in AASV in stable remission as well as in different degrees of activity. Our main objectives were: (1) to find clues to the pathogenesis of these diseases and (2) to discern specific cytokine patterns associated with the prognosis. In an earlier study we found raised circulating levels of IL-6 in combination with normal levels of sTNFr1. ${ }^{14}$ Raised IL-6 levels seemed to be associated with poor prognosis and increased relapse tendency. Here we have chosen to look further into IL-6-levels in plasma, together with the neutrophil chemoattractant IL-8, the monocyte chemoattractant MCP-1, the monocyte activation marker neopterin and the more anti-inflammatory cytokine IL-10. We have also studied the mRNA expression of IL-6, IL-8 and IL-10 in peripheral blood monocytes isolated from 19 patients with AASV in different phases.

\section{Methods}

\section{Patients}

Cohort 1 consisted of 59 patients with AASV, according to the Chapel Hill Consensus Conference disease 
definition, that were included in a cross-sectional study in 1996. The patients were then followed clinically for 6 years, as has been described earlier. ${ }^{15}$ The main focus in this study is on cohort 2 (Table 1). Between February 2001 and March 2003, every patient with defined AASV that came to our clinic was invited to be included. Our exclusion criteria were dialysis treatment, bacterial or viral infections and cancer. Based on clinical observations performed by their regular doctors at the Department of Nephrology, Lund University Hospital, the patients' initial status was classified as remission (Birmingham Vasculitis Activity Score [BVAS] 0-1), chronic grumbling activity (BVAS $<5$ ) or new activity. The latter group was subdivided into minor (BVAS $<5$ ) or major relapse $($ BVAS $>5)$. The patients were monitored during 2 years at their regular visits to the clinic. Clinical status, BVAS, relapses as well as the development of any severe organ damage (cerebrovascular catastrophe, acute myocardial ischemia, renal failure, subglottic stenosis), started dialysis or death due to vasculitic complications was registered. These observations were made without access to the results of our analyses. The patients were grouped according to ANCA specificity (PR3 or MPO, PR3+ $\mathrm{MPO}$, seronegative) as well as development of severe organ damage or not, and relapse within 3 months of sampling or not.

In principle, the data presented in tables and figures are based on the index sample of each patient. However, all relapse samples and samples from patients who developed relapse within 3 months (index samples as well as later samples) have been included. Without this enrichment, the groups would have been too small to compare. There is only one sample from each patient in each group. However, due to the enrichment already described, different samples from the same patient can, very occasionally, occur in different groups. The data from the longitudinal analyses are not shown due to simplicity, but are described in the text. Our control group consisted of 30 healthy blood donors (HBD). From 19 patients with AASV and 11 HBD a blood sample was taken for isolation of peripheral blood monocytes and RNA extraction. These studies were performed with the permission of the Ethical
Committee, Lund University and the written informed consent of the patients.

\section{Blood samples}

Twelve milliliters of venous blood was obtained from each subject in ethylenediamine tetraacetic acid (EDTA) tubes and centrifuged within $1 \mathrm{~h}$. Plasma was carefully aspirated with a Pasteur pipette and stored at $-20^{\circ} \mathrm{C}$ until assayed. For monocyte isolation, $48 \mathrm{ml}$ of venous blood from each subject was obtained in EDTA tubes. The blood was kept at $4{ }^{\circ} \mathrm{C}$. First, $36 \mathrm{ml}$ was immediately taken and put into monocyte purification. The remaining $12 \mathrm{ml}$ were centrifuged within $1 \mathrm{~h}$ and plasma aspirated.

\section{IL-6, IL-8, IL-10 and MCP-1}

A quantitative sandwich enzyme immunoassay from R\&D systems (Abingdon, UK), where a monoclonal antibody specific for IL-6, IL-8, IL-10 or MCP-1 had been pre-coated onto a microplate, was used. Assay Diluent RD1A and Standard or sample was added to each well and left to incubate for $2 \mathrm{~h}$ at room temperature. The plates were washed four times to eliminate any unbound substances. Then conjugate (a polyclonal antibody conjugated to horseradish peroxidase) was added to each well for detection of the cytokine. After $2 \mathrm{~h}$ incubation at room temperature, the plates were washed four times and substrate solution was added to each well. Twenty minutes of incubation at room temperature allowed color development in proportion to the amount of cytokine bound in the initial step. Finally, stop solution was added to each well and the intensity of the coloring measured. The absorbance was read at $450 \mathrm{~nm}$ and $570 \mathrm{~nm}$ (correction wavelength).

\section{Neopterin}

A competitive enzyme immunoassay from IBL Hamburg (Hamburg, Germany), where a peroxidaseconjugated antigen competes with a non-conjugated antigen for a fixed number of antibody-binding sites, was used. The enzyme-linked immunosorbent assay plates had been pre-coated with goat-anti-rabbit

Table 1. Patient characteristics

\begin{tabular}{|c|c|c|c|c|c|}
\hline & Remission & Grumbling & New activity & Minor & Major \\
\hline Patients $(n)$ & 68 & 26 & 21 & 10 & 11 \\
\hline MPO/PR3/MPO + PR3/seronegative & $19 / 43 / 1 / 5$ & $9 / 14 / 1 / 2$ & $5 / 13 / 1 / 2$ & $2 / 6 / 1 / 1$ & $3 / 7 / 0 / 1$ \\
\hline Females/males & $29 / 39$ & $14 / 12$ & $8 / 13$ & $5 / 5$ & $3 / 8$ \\
\hline Age (range) (years) & $63(19-88)$ & $65(26-82)$ & $60(26-83)$ & $59(31-83)$ & $61(26-82)$ \\
\hline Relapse within 3 months $(n)$ & 6 & 5 & - & - & - \\
\hline Organ damage $(n)$ & 12 & 5 & 5 & 1 & 3 \\
\hline
\end{tabular}

Grumbling, chronic smoldering activity; minor, new smoldering activity; major, acute relapse; MPO, anti-myeloperoxidase-ANCA-positive patients; PR3, anti-proteinase 3-ANCA-positive patients; seronegative, ANCA-negative WG; relapse, new or worsened disease activity; organ damage, severe organ damage, start in dialysis or death due to vasculitic complications. 
antibodies. Standard, controls or samples were added together with enzyme-conjugated neopterin and rabbit neopterin antiserum and left to incubate for 90 min at room temperature. After washing, substrate solution was added and finally, after $10 \mathrm{~min}$ incubation, the stop solution was added. Absorbance was measured at $450 \mathrm{~nm}$ and $570 \mathrm{~nm}$ (correction wavelength). The optical density was inversely proportional to the neopterin concentration of the sample.

\section{Cystatin C, C-reactive protein and white blood cell}

Cystatin $\mathrm{C}$ was measured as a marker of glomerular filtration. ${ }^{16} \mathrm{C}$-reactive protein (CRP) is a conventional marker of inflammation.

The Clinical Chemical Laboratory at Lund University Hospital, Lund, Sweden performed analyses on a Hitachi 917 Pluto (Hitachi, Tokyo, Japan). Kits from Roche Diagnostics (Basel, Switzerland) and Dako were used (Glostrup, Denmark).

\section{ANCA}

Wieslab AB (Lund, Sweden) performed analyses of PR3-ANCA and MPO-ANCA by routine methods. ${ }^{17}$

\section{Cell separation}

Peripheral blood monocytes were isolated by means of a monocyte isolation technique based on the OptiPrep density-gradient medium (Axis-Shield PoC AS, Oslo, Norway). ${ }^{18}$ The method is carried out at $4{ }^{\circ} \mathrm{C}$, under sterile conditions, using sterile solutions. Briefly, OptiPrep working solution was added to whole blood. A centrifugation gradient was created by mixing OptiPrep working solution and solution B (Dubecco's modified Eagle's medium cell medium with 10\% serum; Invitrogen, Carlsbad, CA, USA). Five milliliters of blood was pipetted into a $15 \mathrm{ml}$ test tube, after that a $5 \mathrm{ml}$ gradient was carefully added and, finally, $0.5 \mathrm{ml}$ of solution B on top (in order to avoid banding of the cells at the liquid-air interface). During the subsequent centrifugation $(700 \times g$, $30 \mathrm{~min}, 4^{\circ} \mathrm{C}$, no brake during deceleration) the monocytes float to the top of the gradient layer. After collection, the cells were gently diluted with 2 vol of solution $\mathrm{B}$, harvested by centrifugation and resuspended in solution B. Then $25 \mu$ of cell suspension was mixed with Türk's solution (methyl-violet) and counted in a Bürker chamber. A cell smear from each sample was also stained with May-Grünewald for differential counting. The monocyte purity was 80-85\%, with 15-25\% lymphocytes.

\section{RNA extraction}

Total RNA was extracted with the RNeasy Mini kit (VWR International, West Chester, PA, USA) using the supplied protocol. Briefly the monocytes were homogenized and lyzed in the presence of a denaturing guanidine isothiocyanate-containing buffer, which immediately inactivates RNases. Then $70 \%$ ethanol is added to provide appropriate binding conditions, and the sample is then added to an RNeasy mini column with a silica-gel membrane, which binds total RNA. After washing, RNA is eluted in RNase free water. High purity and good integrity were determined in two ways. First, by optical density, $260 / 280 \mathrm{~nm}$ spectrophotometric ratios, and then by the Agilent 2100 Bioanalyzer, using the RNA 6000 Nano Assay reagent kit (Agilent Technologies, Palo Alto, CA, USA). After adding a gel-dye mix together with the RNA sample (25-500 ng) to the RNA 6000 Nano Chip channel system, the bioanalyzer uses electrophoretic and electroosmotic forces to drive fluids through capillaries to produce a virtual gel image and an electropherogram. In the electropherogram, RNA of good quality appears with clear $18 \mathrm{~S}$ and $28 \mathrm{~S}$ rRNA peaks and a flat baseline, whereas in the gel you see the corresponding sharp bands the larger ribosomal band being more intense. Only RNA samples that met these criteria were accepted for further analyses.

\section{Quantitative polymerase chain reaction assay}

Total RNA was transcribed into cDNA, using the Taqman Reverse Transcription Reagents kit (Applied Biosystems, Foster City, CA, USA), according to the manufacturer's recommendations. Briefly, random hexamers were used as template and put into the mastermix together with MultiScribe Reverse Transcriptase, RNase inhibitor, dNTPs, $\mathrm{MgCl}_{2}$ and reverse transcription buffer. Then $500 \mathrm{ng}$ total RNA was added in each $50 \mu \mathrm{l}$ reaction. For determination of gene expression, quantitative polymerase chain reaction assays were performed on an ABI PRISM 7000 Sequence Detector (Applied Biosystems) with Taqman Universal Master Mix UNG, using the standard conditions determined by the company. Assay on Demand, a unique combination of forward and reverse primers and fluorescent MGB probes designed by the company, was used for each target gene. $\beta$-Actin expression levels were used for normalization. Ten nanograms of RNA was used per $25 \mu \mathrm{l}$ reaction and each reaction was performed in triplicate. The level of expression was calculated based upon the polymerase chain reaction cycle number $(\mathrm{Ct})$ at which the exponential growth in fluorescence from the probe passes a certain threshold value. Relative expression was determined by the difference in the Ct values for the target genes after 
normalization to RNA input level, using $\beta$-actin $\mathrm{Ct}$ values. Relative quantification was determined by standard $2^{(-\Delta \Delta \mathrm{Ct})}$ calculations. ${ }^{19}$ Data are presented in relation to the median value of the HBD, set as 1 .

\section{Statistical analysis}

All statistics were performed in StatView 5.01. For correlation analysis, the non-parametric Spearman rank correlation test was used in order to reduce the impact of outliers. Analysis of variance was carried out using the non-parametric Kruskal-Wallis test and the Mann-Whitney U-test.

\section{Results}

\section{Interleukin-6}

In the cross-sectional study we found elevated levels of IL-6 in AASV in remission. ${ }^{15}$ During the 6-year follow-up period, 26 out of 59 patients experienced relapse and 24 patients developed severe organ damage. Patients who subsequently developed relapses had as a group somewhat raised concentrations compared with those who did not (median values, $5.7 \mathrm{ng} / \mathrm{l}$ versus $2.8 \mathrm{ng} / \mathrm{l}, p<0.05$ ). Patients who developed organ damage had a median IL-6 of $4.4 \mathrm{ng} / \mathrm{l}$, compared with $2.0 \mathrm{ng} / \mathrm{l}$ for those who did not $(p<0.05)$. Some correlation was seen with cystatin $\mathrm{C}$, indicating decreased renal elimination. The differences were, however, to some extent maintained after division by cystatin $\mathrm{C}$. The ANCA titer did not seem to matter, except that in patients with MPO-ANCA the IL-6 levels and prognosis tended to have stronger correlation than in patients with PR3-ANCA, especially after correction for decreased renal function. These findings prompted us to start a longitudinal study.

All samples collected in the longitudinal study as presented in Table 1 were analyzed for IL-6. We found significantly raised circulating levels of IL-6 in patients with AASV in the active phase as well as in remission compared with HBD $(p<0.0001)$ (Table 2 and Fig. 1). Patients in remission and patients with grumbling disease activity or minor relapses had similar IL-6 levels. Patients with major relapses, on the other hand, had significantly higher plasma levels $(p<0.001)$. Patients in the stable phase (either remission or grumbling activity) with severe organ damage during the follow-up period had significantly raised IL-6 levels (4.8 ng/l [1.3-18.8] versus $2.6 \mathrm{ng} / \mathrm{l}$ [undetermined-34.3], $p<0.05$; Fig. 2). Also, when looking at patients who did or did not develop relapse within 3 months from sampling, IL-6levels were significantly higher in the former group (5.3 ng/l [2.1-17.5] versus $2.6 \mathrm{ng} / \mathrm{l}$ [undetermined31.8], $p<0.05$; Fig. 3). Repeated measurements

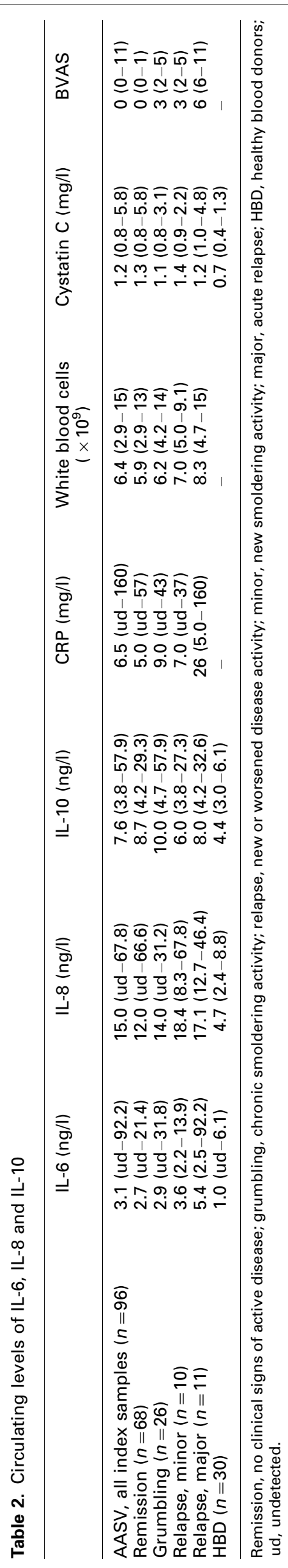




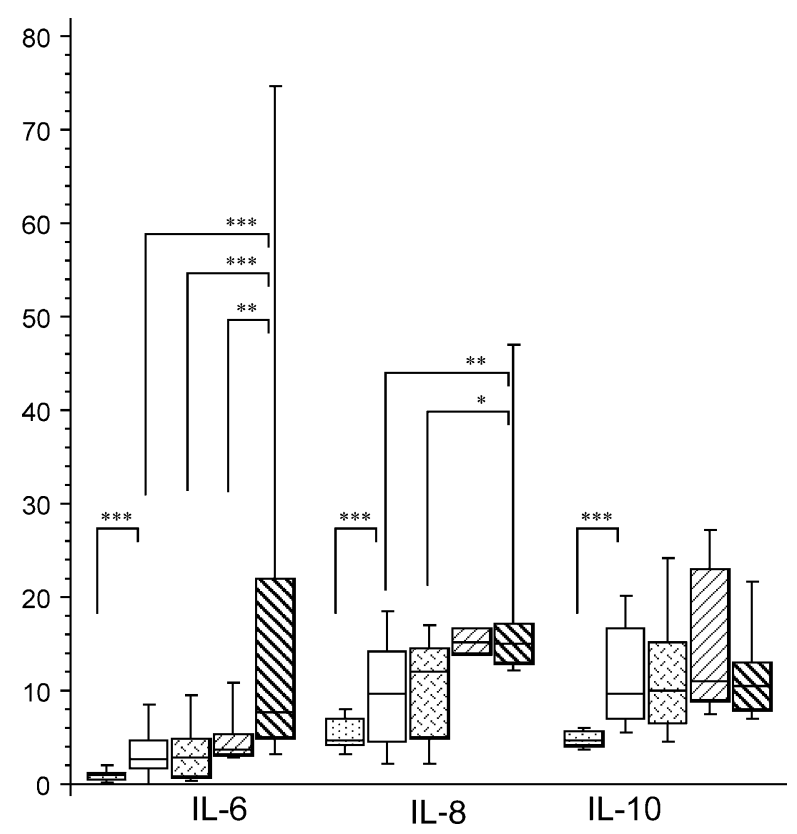

FIG. 1. Circulating cytokine levels from patients with ANCAassociated vasculitis, separated according to disease activity. $\square$, results from patients in remission; 图, results from patients with grumbling disease; $\mathbb{Z}$, patients with minor relapse; $\mathbb{N}$, patients with major relapse; 1 , healthy control group. ${ }^{*}, *$, $* * *$ Significant $p$ values, relative to controls $(p<0.05$, $p<0.01, p<0.001$, respectively) when using the MannWhitney U-test.

showed intra-individual variation, somewhat correlating to disease activity but with a tendency of rising IL-6 levels prior to relapse. Just like in the crosssectional study, a positive correlation was seen between IL-6 and cystatin C (Table 3). After division by cystatin $\mathrm{C}$, the raised IL-6 levels in patients who

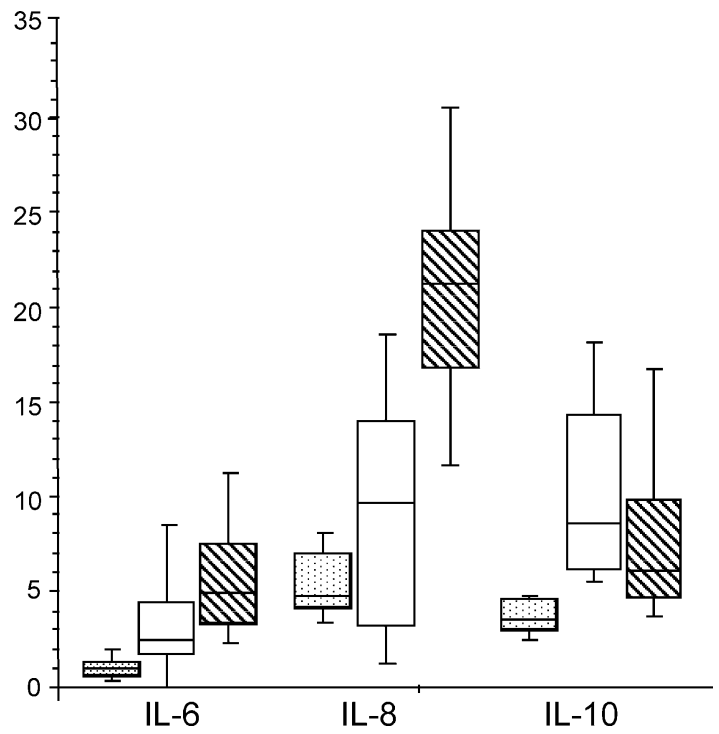

FIG. 2. Circulating cytokine levels in vasculitis patients in stable phase, separated according to subsequent development of severe organ damage during the follow-up period. $\mathbb{N}$, results from 17 patients who did develop organ damage during the follow-up period; - , results from 38 patients were no adverse event was observed (for 77 IL-6 patients); healthy control group.

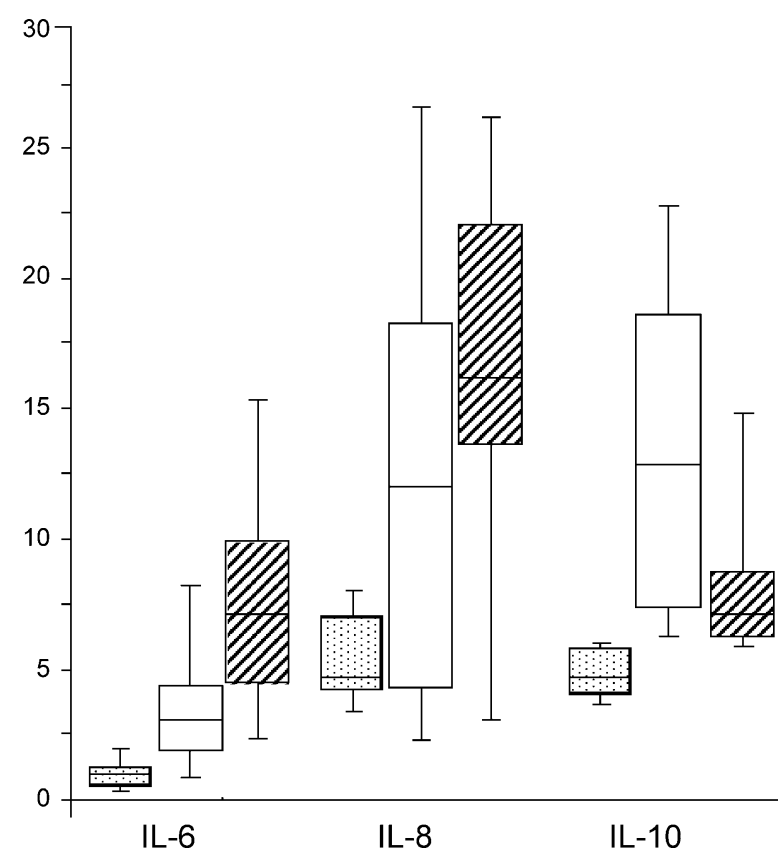

FIG. 3. Circulating cytokine levels from vasculitis patients in the stable phase, separated according to subsequent development of overt relapse. $\mathbb{N}$, results from 11 patients who did relapse within the following 3-month period; -, results from 40 patients where no relapse was detected for at least 3 months (for 80 IL-6 patients); 范, healthy control group.

developed organ damage (renal damage was the most common organ damage) was no longer significant. This phenomenon argues against IL-6 as an important mediator of organ damage, although this cannot be ruled out completely. ${ }^{20}$ IL-6 as a predictor of relapse was, however, strengthened by cystatin $\mathrm{C}$ division $(p<0.001$ compared with $p<0.05)$, indicating a pathogenic role. The other differences described were maintained even after division by cystatin C. ANCA titer and specificity had no obvious connection with the IL-6 levels, whereas the correlation between IL- 6 and CRP was strong (Table 3 ). The raised IL-6 levels shown here are moderate, since IL-6 in sepsis reaches $200-2000 \mathrm{ng} / \mathrm{l}^{21}$

\section{Interleukin-8}

One-half of the samples from the longitudinal study were randomly subjected to additional analyses. As shown in Fig. 1, IL-8 was significantly elevated in all patient groups, compared with $\operatorname{HBD}(p<0.05)$. The levels were higher in patients with relapse, regardless of severity, than in patients in stable phase $(p<0.05)$ (Table 2). Patients in the stable phase (either remission or grumbling activity) who developed organ damage during the follow-up had higher levels of IL8 than those who did not $(21.5 \mathrm{ng} / 1$ [11.5-66.6] versus $9.8 \mathrm{ng} / \mathrm{l}$ [undetermined-4.6], $p<0.0001$; Fig. 2). In the group that developed relapse within 3 months, elevated IL-8 levels were seen compared with those who did not (16.4 ng/1 [undetermined-28.8] versus 
Table 3. Correlation matrix

\begin{tabular}{|c|c|c|c|c|c|c|c|c|}
\hline & BVAS & ANCA & Cystatin C & CRP & Neopterin & MCP-1 & IL-10 & IL-8 \\
\hline IL-6 & $0.2, \mathrm{~ns}$ & $0.2, \mathrm{~ns}$ & $0.4 * * *$ & $0.5 * * *$ & $0.5^{*}$ & $0.5^{*}$ & $-0.1, \mathrm{~ns}$ & $0.3^{*}$ \\
\hline IL-8 & 0 & $-0.3, \mathrm{~ns}$ & $0.4^{*}$ & $0.2, \mathrm{~ns}$ & $0.4, \mathrm{~ns}$ & $0.5^{*}$ & 0 & \\
\hline IL-10 & $0.1, \mathrm{~ns}$ & $0.2, \mathrm{~ns}$ & 0 & 0 & $-0.2, \mathrm{~ns}$ & $-0.2, \mathrm{~ns}$ & & \\
\hline MCP-1 & 0 & $0.1, \mathrm{~ns}$ & $0.7 * * *$ & 0 & $0.6, \mathrm{~ns}$ & & & \\
\hline Neopterin & $0.4, \mathrm{~ns}$ & $0.2, \mathrm{~ns}$ & $0.7 * * *$ & $0.3, \mathrm{~ns}$ & & & & \\
\hline
\end{tabular}

The correlations are expressed as Rho: ${ }^{*} p<0.05,{ }^{*} p<0.001,{ }^{* *} p<0.0001$; ns, not significant, $p>0.05$, using the non-parametric Spearman correlation test. $0=$ Rho $<0.1$.

$12.1 \mathrm{ng} / \mathrm{l}$ [undetermined-66.6]), although this was not statistically significant (Fig. 3). Repeated measurements showed intra-individual variation, correlating to some extent with clinical disease activity. IL-8 showed no correlation with either CRP or ANCA titer and only a weak correlation with cystatin C (Table 3 ). Nor did ANCA specificity seem to matter. For comparison, IL-8 reaches $200-2000 \mathrm{ng} / 1$ in sepsis. ${ }^{22}$

\section{Interleukin-10}

IL-10 was analyzed in the same set of samples as IL-8. As shown in Fig. 1, IL-10 levels were virtually the same, regardless of disease activity (Table 2). No difference was seen between IL-10 levels in patients in the stable phase (either remission or grumbling activity) who did or did not develop organ damage during follow-up (Fig. 2). As shown in Fig. 3, patients who experienced relapse within 3 months of sampling tended to have lower levels of circulating IL-10 than the other patients (7.0 ng/1 [5.6-15.5] versus $13.0 \mathrm{ng} / 1$ [4.4-57.9], $p<0.05)$. Repeated measurements showed some intra-individual variation, irrespective of disease activity. Prior to relapse, five out of 10 patients had persistently lower IL-10 values, three fell and two rose. Just like IL-8, IL-10 showed no correlation with either CRP or ANCA titer (Table 3). Nor did renal function or ANCA specificity seem to matter. IL-10 has in sepsis been reported increased to around $20 \mathrm{ng} / \mathrm{l}^{22}$ A recently published study, however, showed low IL-10 levels (median, $1.5 \mathrm{ng} / \mathrm{l}$; range, $0.1-6.9 \mathrm{ng} / \mathrm{l}$ ) in critically ill patients with sepsis development. ${ }^{23}$

\section{MCP-1}

The monocyte chemoattractant MCP-1 was analyzed in the same set of samples as IL-8 and IL-10. No elevation was seen in patients with AASV in the stable phase compared with HBD (157 ng/1 [54-345] versus $153 \mathrm{ng} / \mathrm{l}$ [72-295]). Nor were there any increased values in patients with relapse. No differences were found regarding development of organ damage and relapses or not. Strong positive correlation was seen between MCP-1 and cystatin C, indicating an influence of decreased renal filtration. As is also presented in Table 3, no correlation was seen with either CRP or ANCA titer.

\section{Neopterin}

Neopterin is a marker of monocyte activation, ${ }^{24}$ measured in 40 of the samples in the longitudinal study. It was significantly raised in AASV in the stable phase, compared with HBD (8.0 nmol/1 [4.5-13.7] versus undetected), but no difference was seen between different degrees of activity. Nor was there any difference with regard to development of organ damage or relapses. What we did find, as presented in Table 3, was a very strong positive correlation with cystatin C. As is also presented in Table 3, no correlation was seen with either CRP or ANCA titer. Taken together, the raised neopterin levels did not seem to depend on increased monocyte activation, but rather on decreased elimination.

\section{sTNFr1}

Another marker of monocyte activity and also of the TNF/TNF-receptor system is sTNFr1. ${ }^{25}$ This protein was measured in the cross-sectional study (cohort 1) and was found to be significantly elevated in AASV compared with HBD (2.9 $\mu \mathrm{g} / 1$ [0.9-23.9] versus $1.1 \mu \mathrm{g} / 1[0.8-2.1], p<0.05)$. Just as for neopterin, the elevated levels could, however, be explained by decreased renal function. Cystatin $\mathrm{C}$ correlated strongly with sTNFr1 $(r=0.5, p<0.0001)$, and after division by cystatin $\mathrm{C}$ there was no difference between AASV and HBD. ${ }^{15}$

\section{Monocytes as a potential source}

Considering monocytes as a potential source of the upregulated cytokines IL-6, IL-8 and IL-10, peripheral blood monocytes were purified from 19 patients with AASV and $11 \mathrm{HBD}$. Twelve patients were in stable remission, five patients had chronic grumbling activity and two patients had acute relapse. All data were compared with and expressed in relation to the median HBD, set as standard $(=1)$. IL-6-expression tended to be lower in AASV than in HBD, but the expression levels were overall very low and data therefore too uncertain to interpret, but contradicting an unspecific activation of the cells during the purification process. As for IL-8 and IL-10, the patients demonstrated somewhat increased expression compared with HBD (Table 4), although the 
Table 4. Monocyte expression of IL-8 and IL-10

\begin{tabular}{lll}
\hline & $\begin{array}{l}\text { IL-8 mRNA } \\
\text { expression }\end{array}$ & $\begin{array}{c}\text { IL-10 mRNA } \\
\text { expression }\end{array}$ \\
\hline AASV $(n=19)$ & $2.8(0.2-36)$ & $2.2(0.4-8.8)$ \\
MPO-positive $(n=6)$ & $6.0(1.0-17)$ & $2.2(0.8-5.6)$ \\
PR3-positive $(n=12)$ & $1.9(0.2-36)$ & $2.2(0.4-8.8)$ \\
Seronegative $(n=1)$ & 2.0 & 0.9 \\
HBD $(n=11)$ & $1.0(0-230)$ & $1.0(0.3-4.0)$ \\
\hline
\end{tabular}

MPO-positive, patients with positive MPO-ANCA; PR3-positive, patients with positive PR3-ANCA. The real-time data are corrected according to the $2^{(-\Delta \Delta C t)}$ formula and are then expressed in relation to the median value of the healthy controls. Results are presented as median (range).

differences were not significant. There was a positive correlation $\left(r^{2}=0.3\right)$ between circulating levels of IL8 and monocyte IL-8 mRNA levels, although nonsignificant (Fig. 4). No such correlation was seen for IL-10. Thus monocytes are a potential source of circulating IL-8 and perhaps also IL-10 in AASV, whereas IL-6 seems to have a different origin.

\section{Discussion}

AASV is characterized by relapsing-remitting inflammation in the small vessels, and prominent infiltrates of neutrophils and monocytes are seen in the vessel wall. ${ }^{1}$ Although there is a strong association with the presence of $\mathrm{ANCAs},{ }^{2}$ the pathogenic role of these antibodies has not yet been completely delineated. Some patients have persisting high ANCA titers when in clinical remission, indicating that ANCA alone is not enough to induce disease. In an earlier study, we found raised circulating levels of IL-6, which to some extent were associated with poor prognosis and increased relapse tendency. ${ }^{14}$ In this study, our aim

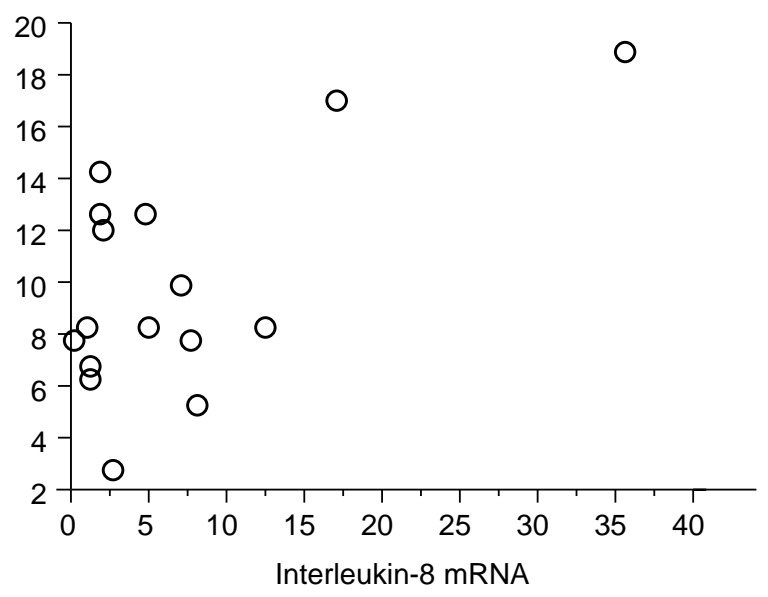

FIG. 4. Correlation between plasma concentration and monocyte mRNA levels from 19 patients with ANCA-associated vasculitis with different disease activity. Plasma levels were measured ( $\mathrm{ng} / \mathrm{l}$ ) using an enzyme-linked immunosorbent assay. mRNA levels are normalized using $\beta$-actin as house keeping gene and the median value of the healthy controls is set to 1.0 . The correlation coefficient is 0.374 , nonsignificant. was to further elucidate the cytokine pattern in the circulation of patients with AASV. Our intention, in the long run, is to find specific patterns with prognostic value and to discern pathogenic clues. In this study we also investigate the monocyte as a potential cytokine source, exploring its role as a central character in the scheme of pathogenesis. Our main finding is that circulating cytokine patterns in patients with ANCA-associated vasculitis in stable remission differ compared with healthy controls. Even during remission patients have raised circulating levels of IL-6, IL-8 and IL-10. High IL-8 levels seem to be associated with poor prognosis, whereas lower levels of IL-10 and higher levels of IL-6 could herald a greater risk of relapse.

Earlier studies within this field are relatively scarce and have largely focused on patients with acute exacerbation. ${ }^{3-6}$ In a recently published study by Wikman et al. ${ }^{26}$ plasma as well as monocytes from patients with newly diagnosed acute anti-PR3-positive vasculitis was compared with patients with acute infection and healthy controls. Increased plasma levels of IL-6, sTNFr1, IL-10 and IL-8 were shown in both disease groups, whereas MCP-1 levels were similar to those in healthy controls. The monocytes showed a reduced capacity to produce oxygen radicals, which together with lower circulating levels of sCD14 and IL-6 than in patients with acute infection, could be a consequence of prolonged immune activation. This would be well in line with our findings, showing that even patients in stable remission have significantly elevated plasma levels of IL-6, IL-8 and IL-10, within the same range as patients who have chronic, grumbling activity. The patients usually have varying doses of immunosuppressants, which could affect the results. In this study 25 samples from patients with no immunosuppressive treatment at all were included and did not differ from the rest in our analyses (data not shown). Decreased renal filtration could have some influence on IL-6 levels, whereas IL-8 and IL-10 did not seem to be affected.

We also wanted to explore potential variations in cytokine expression profile in relation to patient outcome. High IL-6 levels were associated with development of permanent organ damage (although non-significant after correction for decreased renal filtration) as well as relapse tendency $(p<0.001)$. Many have implicated IL-6 in the development of atherosclerosis. ${ }^{27}$ The finding of persisting high levels of circulating IL-6 is therefore consistent with an increased risk of atherosclerotic complications in these patients. ${ }^{28}$ IL-10, on the other hand, has a counterbalancing capacity and the IL-10 genotype is predictive of the risk of cardiovascular disease in end-stage renal disease. ${ }^{29}$ In this context, possible chronic monocyte activation is of course of interest, 
considering the central role of monocytes in the development of atherosclerotic plaques. ${ }^{28}$

Monocyte activation in AASV has mainly been attributed to the influence of ANCA, ${ }^{13,26}$ although surface PR3 expression on monocytes, in contrast to that on neutrophils, does not correlate with disease activity. ${ }^{30}$ In our studies we have not been able to confirm any marked monocyte activation. Although increased circulating levels of IL-6, mRNA levels in monocytes were hardly detectable. Furthermore, raised neopterin and STNFr1 levels in plasma were largely attributed to decreased renal filtration and MCP-1 did not differ from healthy controls. Some sort of persisting low-grade activation of the immune system, in accordance with the findings of Wikman et $a l .{ }^{26}$ seems plausible. One possibility is activation of the monocytes upon exit from the blood vessels; another is dysfunctional apoptosis after entrance into surrounding tissue. Other potential sources of course include the lymphocytes, which have been implicated in earlier studies. ${ }^{31}$

The circulating levels of IL-8, a chemokine that has been implicated in the pathogenesis of AASV in a study by Cockwell et al. ${ }^{4}$ were significantly higher in patients with more severe outcome. PR $3^{32}$ can induce IL-8 production in endothelial cells, which is a possibility here, considering the raised circulating levels of PR3 shown in an earlier study. ${ }^{15}$ Monocyte expression of IL-8 mRNA was detected and some correlation was seen with plasma levels.

IL-10 was significantly lower in plasma from patients who subsequently developed a relapse compared with patients who did not, indicating that the counterbalancing properties of IL-10 are necessary in order to retain persistent subclinical disease activity. Two studies have reported polymorphisms in the IL-10 gene associated with WG. ${ }^{33,34}$ This of course makes it very interesting to study the IL-10 genotype in these patients and to identify the source. Monocyte expression of IL-10 mRNA was detected, but no correlation was seen with plasma levels or clinical activity. Neither was there any co-variation between IL-8 and IL-10 mRNA levels

Taken together, our findings indicate that patients with systemic vasculitis in clinical remission have persistent subclinical disease activity, kept under control by inhibitory cytokines. Whether measurements of IL-6, IL-8 or IL-10 could provide useful clinical information or whether IL-10-mimicking drugs would prevent relapses are important questions for the future.

ACKNOWLEDGEMENTS. This study was supported by grants from the Swedish Scientific Council (project 71X-09487), the foundations of the Royal Physiographic Society and Thelma Zoega, the Foundation for Strategic Research, Riksförbundet för Njursjuka, Sweden, and the Medical Faculty, Lund University. Thanks to Lena Gunnarsson, Nermina Jaganjac, Åsa
Pettersson (Department of Nephrology) and Carina Strand (Department of Oncology) for their skilful laboratory assistance.

\section{References}

1. Jennette JC, Wilkman AS, Falk RJ. Anti-neutrophil cytoplasmic autoantibody-associated glomerulonephritis and vasculitis. Am J Pathol 1989; 135: $921-930$.

2. Hellmich B, Csernok E, Gross WL, et al. 20 years with ANCA (antineutrophil cytoplasmic autoantibodies): from seromarker to a major pathogenic player in vasculitis. J Leukoc Biol 2003; 74: 1-2.

3. Noronha IL, Kruger C, Andrassy K, Ritz E, Waldherr R. In situ production of TNF-alpha, IL-1 beta and IL-2R in ANCA-positive glomerulonephritis. Kidney Int 1993; 43: 682-692.

4. Cockwell P, Brooks CJ, Adu D, Savage CO. Interleukin-8: A pathogenetic role in antineutrophil cytoplasmic autoantibody-associated glomerulonephritis. Kidney Int 1999; 55: 852-863.

5. Rastaldi MP, Ferrario F, Tunesi S, Yang L, D'Amico G. Intraglomerular and interstitial leukocyte infiltration, adhesion molecules, and interleukin-1 alpha expression in 15 cases of antineutrophil cytoplasmic autoantibody-associated renal vasculitis. Am J Kidney Dis 1996; 27: $48-$ 57.

6. Arimura Y, Minoshima S, Kamiya Y, et al. Serum myeloperoxidase and serum cytokines in anti-myeloperoxidase antibody-associated glomerulonephritis. Clin Nephrol 1993; 40: 256-264.

7. Segelmark M, Westman K, Wieslander J. How and why should we detect ANCA? Clin Exp Rheumatol 2000; 18: 629-635.

8. Tervaert JW, van der Woude FJ, Fauci AS, et al. Association between active Wegener's granulomatosis and anticytoplasmic antibodies. Arch Intern Med 1989; 149: 2461-2465.

9. Ralston DR, Marsh CB, Lowe MP, Wewers MD. Antineutrophil cytoplasmic antibodies induce monocyte IL-8 release. Role of surface proteinase3, alpha1-antitrypsin, and Fcgamma receptors. J Clin Invest 1997; 100: $1416-1424$

10. Casselman BL, Kilgore KS, Miller BF, Warren JS. Antibodies to neutrophil cytoplasmic antigens induce monocyte chemoattractant protein-1 secretion from human monocytes. I Lab Clin Med 1995; 126: 495-502.

11. Hattar K, Bickenbach A, Csernok E, et al. Wegener's granulomatosis: antiproteinase 3 antibodies induce monocyte cytokine and prostanoid release-role of autocrine cell activation. J Leukoc Biol 2002; 71: $996-$ 1004 .

12. Ferrario F, Rastaldi MP. Necrotizing-crescentic glomerulonephritis in ANCA-associated vasculitis: the role of monocytes. Nephrol Dial Transplant 1999; 14: 1627-1631.

13. Muller Kobold AC, Kallenberg CG, Tervaert JW. Monocyte activation in patients with Wegener's granulomatosis. Ann Rheum Dis 1999; 58: $237-245$.

14. Ohlsson SL, Ohlsson C, Segelmark K, Wieslander M. IL-6 as a potential marker of disease in ANCA-associated systemic vasculitis. 33rd Annual Meeting \& Scientific Exposition of the ASN 2000, 11-16 October, Toronto, Ontario, Canada. Baltimore, MD, USA: JASN.

15. Ohlsson S, Wieslander J, Segelmark M. Increased circulating levels of proteinase 3 in patients with anti-neutrophilic cytoplasmic autoantibodies-associated systemic vasculitis in remission. Clin Exp Immunol 2003; 131: $528-535$.

16. Dharnidharka VR, Kwon C, Stevens G. Serum cystatin C is superior to serum creatinine as a marker of kidney function: a meta-analysis. $A m J$ Kidney Dis 2002; 40: 221-226.

17. Wiik A, Rasmussen N, Wieslander J. Methods to detect autoantibodies to neutrophilic granulocytes. Manual Biol Markers Dis 1993; A9: 1-14.

18. Graziani-Bowering GM, Graham JM, Filion LG. A quick, easy and inexpensive method for the isolation of human peripheral blood monocytes. J Immunol Methods 1997; 207: 157-168.

19. Pendergraft WF, Alcorta DA, Segelmark M, et al. ANCA antigens, proteinase 3 and myeloperoxidase, are not expressed in endothelial cells. Kidney Int 2000; 57: 1981-1990.

20. Pecoits-Filho R, Heimburger O, Barany P, et al. Associations between circulating inflammatory markers and residual renal function in CRF patients. Am J Kidney Dis 2003; 41: 1212-1218.

21. Remick DG, Bolgos GR, Siddiqui J, Shin J, Nemzek JA. Six at six: interleukin- 6 measured $6 \mathrm{~h}$ after the initiation of sepsis predicts mortality over 3 days. Shock 2002; 17: 463-467.

22. Hynninen $M$, Valtonen $M$, Vaara $M$, et al. Plasma interleukin-8, interleukin-10, and E-selectin levels in neutropenic and non-neutropenic bacteremic patients. Eur J Clin Microbiol Infect Dis 1997; 16: $587-$ 591.

23. Lowe PR, Galley HF, Abdel-Fattah A, Webster NR. Influence of interleukin-10 polymorphisms on interleukin-10 expression and survival in critically ill patients. Crit Care Med 2003; 31: 34-38.

24. Berdowska A, Zwirska-Korczala K. Neopterin measurement in clinical diagnosis. J Clin Pharm Ther 2001; 26: 319-329.

25. Diez-Ruiz A, Tilz GP, Zangerle R, Baier-Bitterlich G, Wachter H, Fuchs D. Soluble receptors for tumor necrosis factor in clinical laboratory diagnosis. Eur J Haematol 1995; 54: 1. 
26. Wikman A, Fagergren A, Gunnar OJS, Lundahl J, Jacobson SH. Monocyte activation and relationship to anti-proteinase 3 in acute vasculitis. Nephrol Dial Transplant 2003; 18: 1792-1799.

27. Yudkin JS, Kumari M, Humphries SE, Mohamed-Ali V. Inflammation, obesity, stress and coronary heart disease: is interleukin- 6 the link? Atherosclerosis 2000; 148: 209-214.

28. Ross R. The pathogenesis of atherosclerosis: a perspective for the $1990 \mathrm{~s}$ Nature 1993; 362: 801-809.

29. Girndt M, Ulrich C, Kaul H, Sester U, Sester M, Kohler H. Uremiaassociated immune defect: the IL-10-CRP axis. Kidney Int Suppl 2003 84: $\mathrm{S} 76-\mathrm{S} 79$.

30. Muller Kobold AC, Kallenberg CG, Tervaert JW. Leucocyte membrane expression of proteinase 3 correlates with disease activity in patients with Wegener's granulomatosis. Br J Rheumatol 1998; 37: 901-907.

31. Popa ER, Stegeman CA, Bos NA, Kallenberg CG, Tervaert JW. Differential B- and T-cell activation in Wegener's granulomatosis. J Allergy Clin Immunol 1999; 103: 885-894.
32. Berger SP, Seelen MA, Hiemstra PS, et al. Proteinase 3, the major autoantigen of Wegener's granulomatosis, enhances IL- 8 production by endothelial cells in vitro. J Am Soc Nephrol 1996; 7: 694-701.

33. Murakozy G, Gaede KI, Ruprecht B, et al. Gene polymorphisms of immunoregulatory cytokines and angiotensin-converting enzyme in Wegener's granulomatosis. J Mol Med 2001; 79: 665-670.

34. Zhou Y, Giscombe R, Huang D, Lefvert AK. Novel genetic association of Wegener's granulomatosis with the interleukin 10 gene. J Rheumatol 2002; 29: 317-320

\section{Received 13 May 2004}

Accepted 23 June 2004 


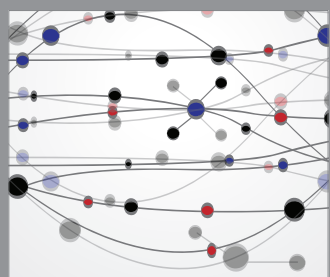

The Scientific World Journal
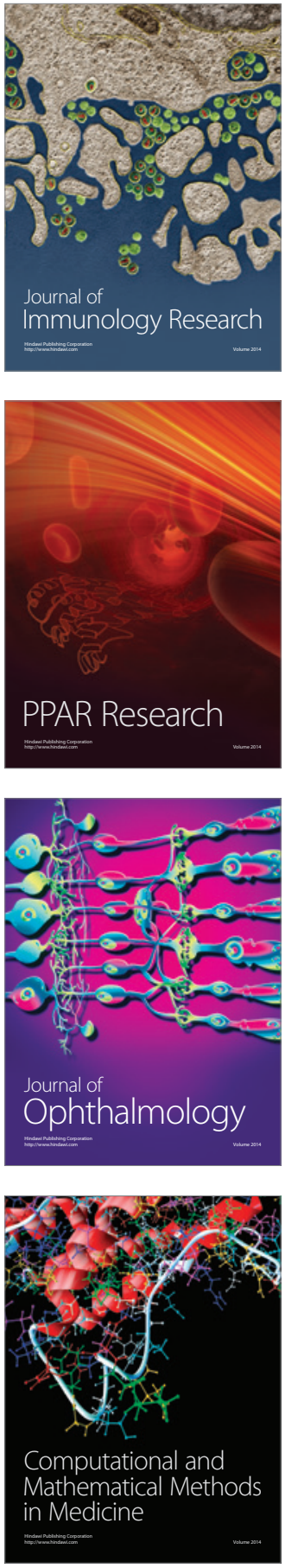

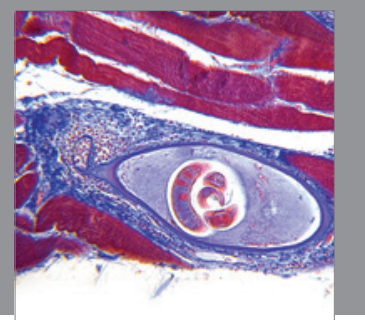

Gastroenterology

Research and Practice
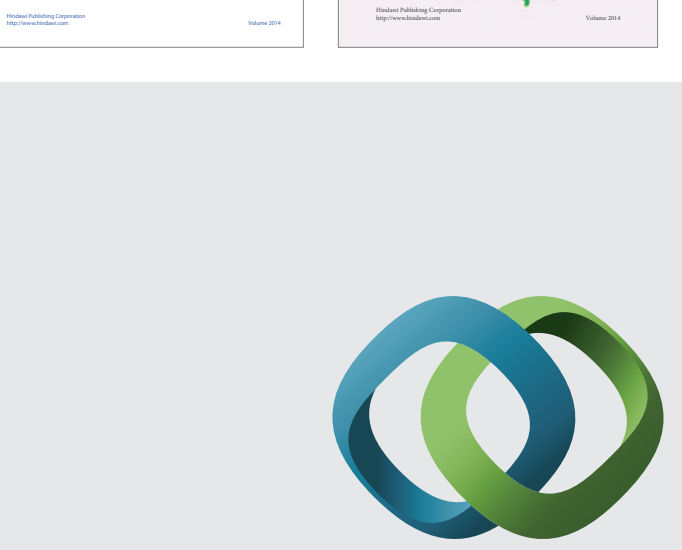

\section{Hindawi}

Submit your manuscripts at

http://www.hindawi.com
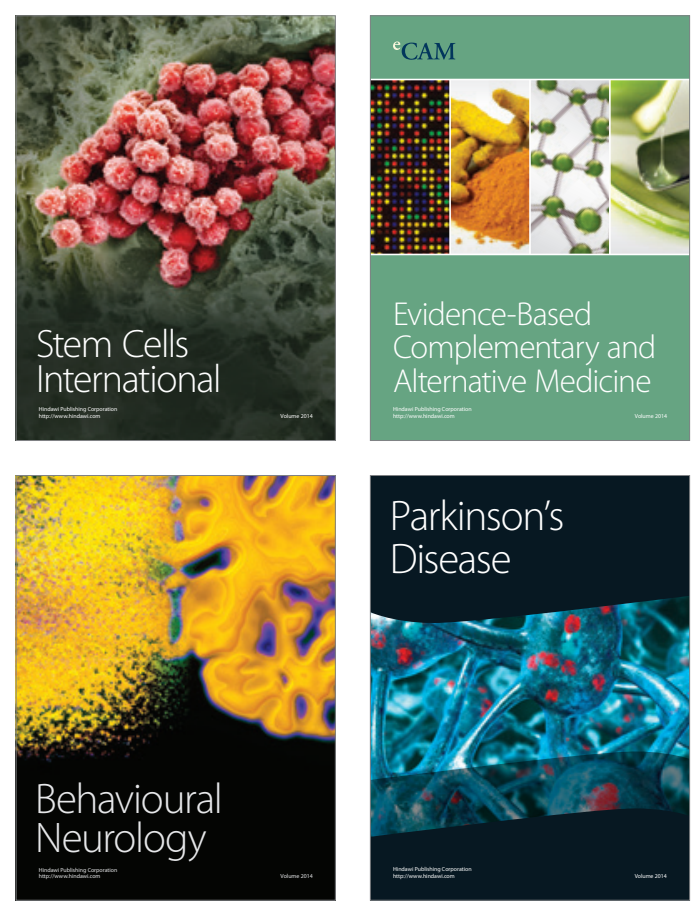

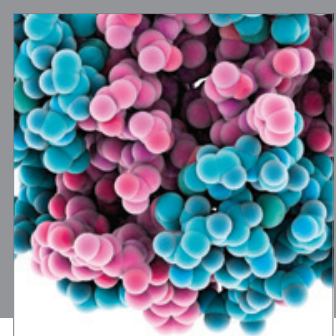

Journal of
Diabetes Research

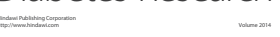

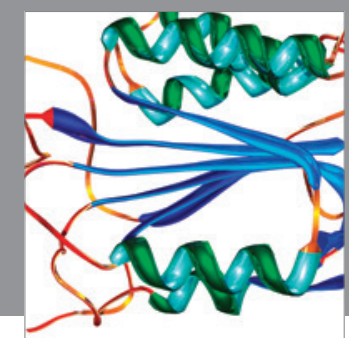

Disease Markers
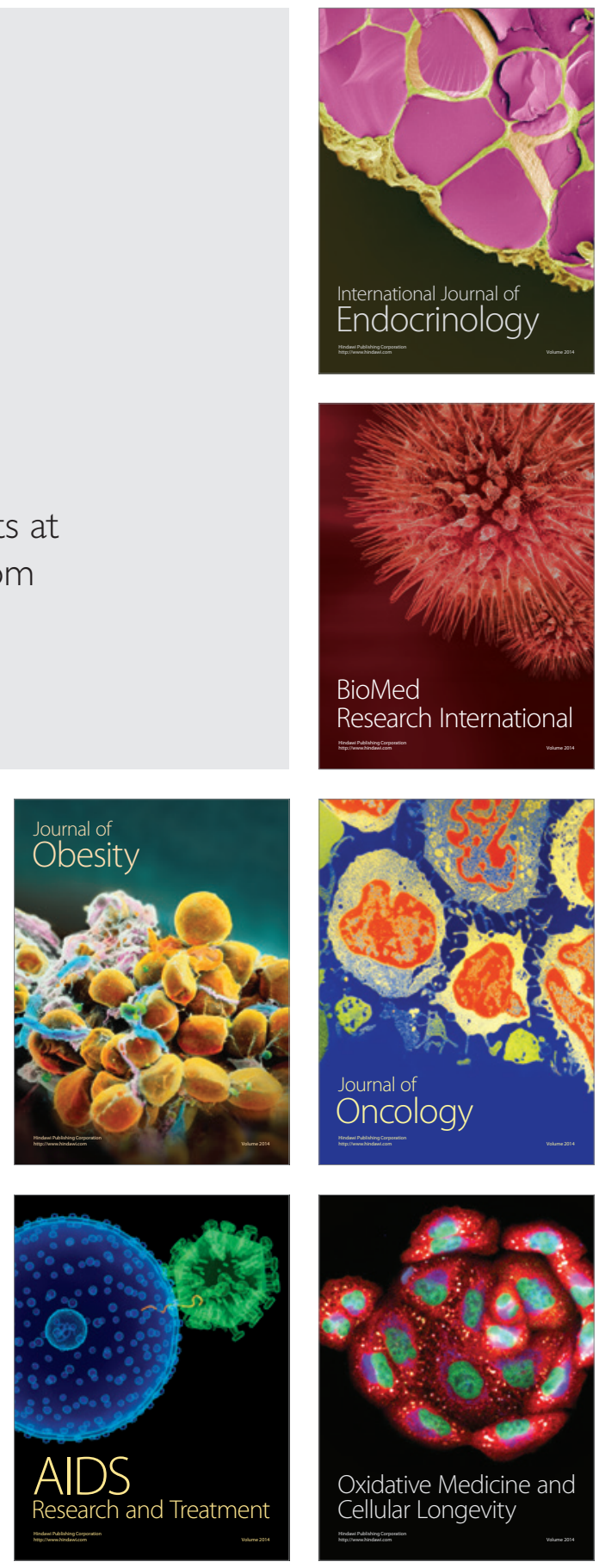